\section{Case Report}

*Corresponding author

Solwayo Ngwenya, MBChB, DFSRH, MRCOG

Consultant Obstetrician and

Gynaecologist

Head of Department of Obstetrics and

Gynaecology, Clinical Director

Mpilo Central Hospital

Vera Road

Mzilikazi Founder and Chief Executive

Officer, Royal Women's Clinic

52A Cecil Avenue, Hillside

Bulawayo, Matabeleland, Zimbabwe

Tel. +2639214965

E-mail: drsolngwe@yahoo.co.uk

Volume 3 : Issue 2

Article Ref. \#: 1000GOROJ3132

\section{Article History}

Received: August $7^{\text {th }}, 2016$

Accepted: August 24 $4^{\text {th }}, 2016$

Published: August 24 ${ }^{\text {th }}, 2016$

\section{Citation}

Ngwenya S. Cryptococcal meningitis in pregnancy, the neglected diagnosis: A case report. Gynecol Obstet Res Open J. 2016; 3(2): 23-25. doi: 10.17140/ GOROJ-3-132

\section{Copyright}

(C2016 Ngwenya S. This is an open access article distributed under the Creative Commons Attribution 4.0 International License (CC BY 4.0), which permits unrestricted use, distribution, and reproduction in any medium, provided the original work is properly cited.

\title{
Cryptococcal Meningitis in Pregnancy, the Neglected Diagnosis: A Case Report
}

Solwayo Ngwenya, MBChB, DFSRH, MRCOG*

Consultant Obstetrician and Gynaecologist, Head of Department of Obstetrics and Gynaecology, Mpilo Central Hospital, Vera Road, Mzilikazi Founder and Chief Executive Officer, Royal Women's Clinic, 52A Cecil Avenue, Hillside, Bulawayo, Matabeleland, Zimbabwe

\section{ABSTRACT}

Introduction: Cryptococcal meningitis is an opportunistic infection of human immunodeficiency virus infection and acquired immune deficiency syndrome (HIV/AIDS) that can cause maternal and fetal mortality and morbidity. This case report aims to help clinicians to consider it as a differential diagnosis in HIV-seropositive pregnant women presenting with vague symptoms. This is particularly important as HIV/AIDS is now a global pandemic. There is a test available for disease surveillance.

Case Report: A 31-year-old P3 G4 HIV seropositive patient on antiretroviral therapy/cotrimoxazole presented at 29 weeks gestation by dates complaining of draining clear fluid per vagina for 24 hours, slight frontal headache and vomiting. On admission, she was ill-looking but was apyrexial with a body temperature of $36.2^{\circ} \mathrm{C}$. A sterile speculum examination confirmed ruptured membranes with a pool of liquor in the posterior vaginal fornix. She was treated as a case of preterm pre-labour rupture of membranes. Therapy consisted of erythromycin and dexamethasone. She complained of increasing frontal headaches and vomiting and on detailed examination was found to be now having neck stiffness. A lumbar puncture was performed and microscopy showed Cryptococcus neoformans. She was managed by a multidisciplinary team involving physicians and was treated with antifungals. She delivered prematurely by normal vaginal delivery a baby boy weighing $2100 \mathrm{~g}$ with Apgar 7 and 9 scores at 1 and 5 minutes respectively. Post-partum she continued to fit for a day but subsequently the convulsions stopped and the patient began to improve clinically.

Conclusion: There is need to raise awareness amongst clinicians about serious opportunistic infections in HIV infected pregnant mothers for early diagnosis and treatment. Antifungal therapy can be life-saving and prevent maternal deaths.

KEYWORDS: Cryptococcal meningitis; Pregnancy; Amphotericin B; Maternal death; Perinatal outcomes.

\section{INTRODUCTION}

The first cases of Cryptococcal neoformans meningitis in pregnancy in the literature were described in $1962^{1}$ and $1972 .{ }^{2}$ It is an opportunistic fungal infection that presents with an insidious onset. Fungal spores like those in Figure 1 can be found in the cerebrospinal fluid in the brain causing significant maternal morbidity and mortality. There is currently sparse information on this subject in the literature. Cryptococcal meningitis is a serious disease that can cause adverse maternal and fetal outcomes especially in HIV/AIDS infected patients. Furthermore, pregnancy is immunosuppressive hence the combination with HIV can be catastrophic with maternal death. The disease can occur in HIV sero-negative and sero-positive patients. ${ }^{3,4}$ The HIV/AIDS pandemic has assumed global status and the cases that occur need to be reported to raise clinical awareness. Awareness among clinicians is vital to help with early diagnosis, treatment and reduce morbidity and mortality. 


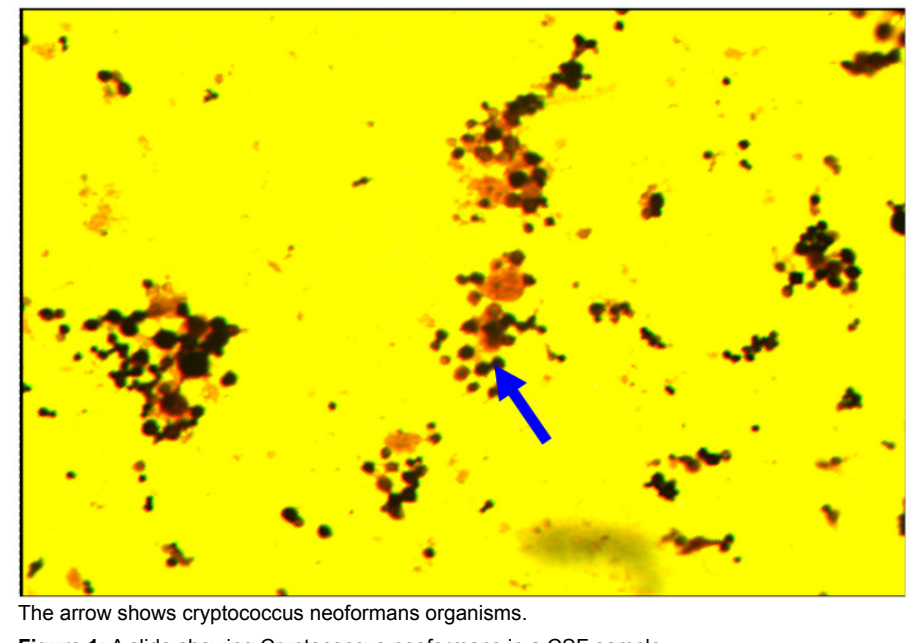

Figure 1: A slide showing Cryptococcus neoformans in a CSF sample.

\section{CASE REPORT}

A 31-year-old P3 G4 HIV seropositive patient on antiretroviral therapy/cotrimoxazole presented at 29 weeks gestation by dates complaining of draining clear fluid per vagina for 24 hours. She also complained of a slight frontal headache and vomiting. She had been feeling dizzy and having generalised body weakness for the past 2 months.

On admission, she was ill-looking but was apyrexial with a body temperature of $36.2^{\circ} \mathrm{C}$. Her blood pressure (BP) recording was $122 / 76 \mathrm{mmHg}$. The fetal heart was normal on Pinard auscultation. A sterile speculum examination confirmed ruptured membranes with a pool of liquor in the posterior vaginal fornix. A high vaginal swab was taken.

The differential diagnosis was that of preterm pre-labour rupture of membranes and urinary tract infection. A full blood count showed a normocytic normochromic anaemia of $9.8 \mathrm{~g} / \mathrm{dl}$, white blood cell (WBC) count of $10.63 \times 10^{9} / \mathrm{L}$, a platelet count of $297 \times 10^{9} / \mathrm{L}$. The CD4 count was 379 cells $/ \mathrm{mm}^{3}$. Urinalysis showed no glucosuria and a trace of protein and a sample was sent for microscopy and culture. An ultrasound scan showed a fetus of 30 weeks gestation and adequate liquor volume. Therapy consisted of Erythromycin $250 \mathrm{mg} 6$ hourly per os to prevent infections and dexamethasone $6 \mathrm{mg}$ intramuscularly twice per day for 2 days for fetal lung maturation. The high vaginal swab and urine samples did not grow any organisms on culture.

After 3 days of admission, she complained of increasing frontal headaches and vomiting. The blood pressures remained within normal ranges. On detailed examination she was found to be now having neck stiffness. A lumbar puncture was done. The cerebrospinal fluid (CSF) was clear but under pressure. CSF glucose and proteins were normal. Microscopic examination showed Cryptococcus neoformans. The culture also grew Cryptococcus neoformans. The serum cryptococcal antigen $(\mathrm{CrAg})$ test was positive.

A team of physicians was consulted and reviewed the patient. The patient was commenced on Amphotericin B $50 \mathrm{mg}$ intravenously once daily plus Fluconazole $800 \mathrm{mg}$ once daily per os. She received dexamethasone $10 \mathrm{mg}$ intravenously 8 hourly.

After 3 weeks of therapy she started having convulsions. The patient went into preterm labour and delivered by normal vaginal delivery a baby boy weighing $2100 \mathrm{~g}$ with Apgar scores 7 and 9 at 1 and 5 minutes respectively. Post-partum she continued to fit for a day but subsequently the convulsions stopped and the patient began to improve clinically. A fortnight after delivery she was completely fit free and asymptomatic and able to sit up and feed properly. The neonate was well, needing no further treatments.

\section{DISCUSSION}

This case report is important in that it raises awareness about an important condition that may cause considerable morbidity and mortality in pregnant patients. Other studies and case reports have reported similar clinical scenarios. This case report therefore adds more knowledge about this condition. Increased awareness can help reduce poor perinatal outcomes.

The differential diagnosis in this patient included preeclampsia/eclampsia but there was no clinical or labarotory evidence to support this diagnosis as the patient had no proteinuria or elevated blood pressures. The other diagnosis to consider was tuberculous meningitis (TBM) but the lumbar puncture results were conclusive for cryptococcal meningitis (CM). HIV/AIDS presentation can present with abnormal ways such as opportunistic infections like cryptococal meningitis.

The prevention of cryptococcal meningitis in subclinical cases in HIV infected patients with CD4 cell counts of $<100$ cells $/ \mathrm{mm}^{3}$ is possible. The diagnostic use of cryptococcal capsular polysaccharide antigen $(\mathrm{CrAg})$ in serum is advisable ${ }^{5}$ this allows life-saving antifungal therapy to be initiated. In low resource settings, this test is not readily available due to cost implications. In the presented case there was delay in diagnosis as 
there were vague clinical symptoms pointing to a serious condition such as meningitis.

The management of the patient should be multidisciplinary involving physicians. Antifungal chemotherapeutic drugs used include Amphotericin B alone or in combination with flucytosine. ${ }^{6}$ Fetal teratogenic effects of these drugs have not been reported, in fact both good maternal and fetal outcomes have been reported. ${ }^{7-11}$ Another antifungal drug that can be used is fluconazole or itraconazole with good outcomes. ${ }^{12}$ Cryptococcal placental infection without neonatal infection has been reported in the literature. ${ }^{13}$ Only one case of mother-to-child transmission of cryptococcosis has been described in the literature. ${ }^{14}$ In this case report, the baby did not seem any signs of being affected hence did not need any further treatments. Patients followed up to 8 months post-infection have shown no signs of infection. ${ }^{15}$

\section{CONCLUSION}

There is need to raise awareness amongst clinicians about serious opportunistic infections in HIV infected pregnant mothers for early diagnosis and treatment. The widespread use of $\mathrm{CrAg}$ as a screening tool can help early disease detection hence allowing the commencement of antifungal therapy that can be life-saving and prevent maternal deaths.

\section{CONSENT}

The authors obtained written informed consent from the patient for submission of this manuscript for publication.

\section{REFERENCES}

1. Aitken GW, Symonds EM. Cryptococcal meningitis in pregnancy treated with Amphotericin B: A case report. J Obstet Gynaecol Br Emp. 1962; 69: 677-679. doi: 10.1111/j.14710528.1962.tb01260.x

2. Philpot CR, Lo D. Cryptococcal meningitis in pregnancy. Med J Aust. 1972; 2(18): 1005-1057. Web site. http://europepmc.org/ abstract $/ \mathrm{med} / 4578877$. Accessed August 6, 2016

3. Nath R, Laskar B, Ahmed J, Das S, Timung L, Saikia L. Cryptococcus neoformans var. grubii infection in HIV-seronegative patients from Northeast India: Report of two cases with review of the literature. Mycopathologia. 2016; 181(3-4): 315-321. doi: 10.1007/s11046-015-9971-5

4. Costa ML, Souza JP, Oliveira Neto AF, Pinto E Silva JL. Cryptococcal meningitis in HIV negative pregnant women: Case report and review of literature. Rev Inst Med Trop Sao Paulo. 2009; 51(5): 289-294. doi: 10.1590/S0036-46652009000500010

5. Vidal JE, Boulware DR. Lateral flow assay for cryptococcal antigen: An important advance to improve the continuum of HIV care and reduce cryptococcal meningitis-related mortality.
Rev Inst Med Trop Sao Paulo. 2015; 57(Suppl 19): 38-45. doi: 10.1590/S0036-46652015000700008

6. Curole DN. Cryptococcal meningitis in pregnancy. $J$ Reprod Med. 1981; 26(6): 317-319.

7. Nayak SU, Talwani R, Gilliam B, Taylor G, Ghosh M. Cryptococcal meningitis in an HIV-positive pregnant women. $J$ Int Assoc Physicians AIDS Care (Chic). 2011; 10(2): 79-82. doi: 10.1177/1545109710387434

8. Stafford CR, Fisher JF, Fadel HE, Espinel-Ingroff AV, Shadomy S, Hamby M. Cryptococcal meningitis in pregnancy. Obstet Gynaecol. 1983; 62(3 Suppl): 35s-37s. Web site. http://journals. lww.com/greenjournal/abstract/1983/09001/cryptococcal_meningitis_in_pregnancy.11.aspx. Accessed August 6, 2016

9. Chotmongkol V, Siricharoensang S. Cryptococcal meningitis in pregnancy: A case report. J Med Assoc Thai. 1991; 74(9): 421-422.

10. Pereira CA, Fischman O, Colombo AL, Moron AF, Pignatari AC. Cryptococcal meningitis in pregnancy: Review of the literature. Report of 2 cases [In Portuguese]. Rev Inst Med Trop Sao Paulo. 1993; 35(4): 367-371.

11. Chen CP, Wang KG. Cryptococcal meningitis in pregnancy. Am J Perinatol. 1996; 13(1): 35-36.

12. Chotmongkol V, Sookprasert A. Itraconazole in cryptococcal meningitis in pregnancy: A case report. J Med Assoc Thai. 1992; 75(10): 606-608.

13. Darko AD, Dim DC, Taylor G, Watson DC, Sun CC. Placental Cryptococcal neoformans infection without neonatal disease: Case report and review of the literature. Paediatr Dev Pathol. 2009; 12(3): 249-252. doi: 10.2350/08-10-0549.1

14. Sirinavin S, Intusoma U, Tuntirungsee S. Mother-to-child transmission of Cryptococcus neoformans. Pediatr Infect Dis $J$. 2004; 23(3): 278-279.

15. Jones JM, Craig WA. Cryptococcal meningitis: Resolution eight months after antifungal therapy. South Med J. 1983; 76(12): 1567-1569. Web site. http://europepmc.org/abstract/ med/6648619. Accessed August 6, 2016 